\title{
Sedimentation velocity and potential in concentrated suspensions of charged porous spheres
}

\author{
Huan J. Keh*, Wei C. Chen \\ Department of Chemical Engineering, National Taiwan University, Taipei 10617, Taiwan, Republic of China \\ Received 24 May 2005; accepted 17 September 2005 \\ Available online 9 January 2006
}

\begin{abstract}
The body-force-driven migration in a homogeneous suspension of polyelectrolyte molecules or charged flocs in an electrolyte solution is analyzed. The model used for the particle is a porous sphere in which the density of the hydrodynamic frictional segments, and therefore also that of the fixed charges, is constant. The effects of particle interactions are taken into account by employing a unit cell model. The overlap of the electric double layers of adjacent particles is allowed and the relaxation effect in the double layer surrounding each particle is considered. The electrokinetic equations which govern the electrostatic potential profile, the ionic concentration (or electrochemical potential energy) distributions, and the fluid velocity field inside and outside the porous particle in a unit cell are linearized by assuming that the system is only slightly distorted from equilibrium. Using a regular perturbation method, these linearized equations are solved for a symmetrically charged electrolyte with the density of the fixed charges as the small perturbation parameter. An analytical expression for the settling velocity of the charged porous sphere is obtained from a balance among its gravitational, electrostatic, and hydrodynamic forces. A closed-form formula for the sedimentation potential in a suspension of identical charged porous spheres is also derived by using the requirement of zero net electric current. The dependence of the sedimentation velocity and potential of the suspension on the particle volume fraction and other properties of the particle-solution system is found to be quite complicated.
\end{abstract}

(C) 2005 Elsevier Inc. All rights reserved.

Keywords: Sedimentation velocity; Sedimentation potential; Charged porous particle; Unit cell model

\section{Introduction}

The sedimentation or migration of charged colloidal particles in electrolyte solutions has received quite an amount of attention in the past. This problem is more complex than that of uncharged particles because the electric double layer surrounding each charged particle is distorted by the ambient fluid flow relative to the particle. The deformation of the double layer resulting from the fluid motion is usually referred to as the polarization or relaxation effect and gives rise to an induced electric field. The sedimentation potential or migration potential, which is set up in a suspension of settling or translating charged particles, was first reported by Dorn in 1878, and this effect is often known by his name [1-5]. The sedimentation potential gradient not only alters the velocity and pressure distributions in the

\footnotetext{
* Corresponding author.

E-mail address: huan@ntu.edu.tw (H.J. Keh).
}

fluid due to its action on the electrolyte ions but also retards the settling of the particles by an electrophoretic effect.

Without considering the particle-particle interaction effects, Booth [1] solved a set of electrokinetic equations using a perturbation method to obtain formulas for the sedimentation velocity and sedimentation potential in a dilute suspension of identical spherical particles with an arbitrary double-layer thickness expressed as power series in the small zeta potential of the particles. A method of thin-double-layer approximation for the evaluation of the sedimentation potential, which has no restriction regarding the value of the zeta potential of the particles, has also been developed $[2,3,6]$. On the other hand, numerical results relieving the restriction of low zeta potential in Booth's analysis were reported by Stigter [7] using a modification of the theory of electrophoresis of a dielectric sphere developed by Wiersema et al. [8]. It was found that the Onsager reciprocal relation between the sedimentation potential and the electrophoretic mobility derived by de Groot et al. [9] is satisfied 
within good computational accuracy. Taking the double-layer distortion from equilibrium as a small perturbation, Ohshima et al. [10] obtained general expressions and presented numerical results for the sedimentation velocity and potential in a dilute suspension of identical charged spheres over a broad range of zeta potential and double-layer thickness.

Theoretical study of the electrokinetic phenomena of charged porous particles, or flocs, was first made by Hermans and Fujita $[11,12]$ who derived formulas for the electrophoretic mobility of a porous sphere by introducing the Brinkman equation $[13,14]$ for the internal flow field of the particle and assuming that the double layer remains spherically symmetric in the presence of the applied electric field. The effect of the distortion of the counterion atmosphere around a nearly free-drained polyelectrolyte coil under an applied electric field was examined theoretically by Imai and Iwasa [15], and their numerical results agree well with the experimental data [16]. Recently, general expressions were derived for the electrophoretic mobility and sedimentation velocity of a charged composite (soft) spherical particle which is a rigid colloidal sphere coated with a layer of porous substances or polymers at its surface [17-22]. These electrophoretic mobility expressions tend to a formula obtained by Hermans and Fujita [11] for a spherical polyelectrolyte when the hard core of the composite particle vanishes and the electric potentials are low.

In practical applications of sedimentation, relatively concentrated suspensions of particles are usually encountered, and effects of particle interactions will be important. To avoid the difficulty of the complex geometry appearing in swarms of particles, unit cell models [23-27] were often employed to predict the effects of particle interactions on the mean sedimentation rate in a bounded suspension of identical spheres. These models involve the concept that an assemblage can be divided into a number of identical cells, one sphere occupying each cell at its center. The boundary value problem for multiple spheres is thus reduced to the consideration of the behavior of a single sphere and its bounding envelope. The most acceptable of these models with various boundary conditions at the virtual surface of the cell are the so-called "free-surface" model of Happel [23] and "zero-vorticity" model of Kuwabara [24], the predictions of which for the sedimentation of uncharged spherical particles have been tested against the experimental data.

Using the Kuwabara cell model and assuming that the overlap of the electric double layers of adjacent particles is negligible on the virtual surface of the cell, Levine et al. [25] derived analytical expressions for the sedimentation velocity and sedimentation potential in a homogeneous suspension of identical charged impermeable spheres with a small surface potential as functions of the fractional volume concentration of the particles. The Kuwabara model with nonoverlapping double layers has also been used to demonstrate the Onsager relation between the sedimentation potential and the electrophoretic mobility of charged impermeable spheres in concentrated suspensions $[28,29]$. On the other hand, the body-force-driven migration phenomena in homogeneous suspensions of identical charged impermeable spheres with a small surface potential and an arbitrary double-layer thickness were analyzed with employing both the Happel and the Kuwabara cell models and allowing the overlap of adjacent double layers [30]. Closed-form formulas for the sedimentation velocity and potential expressed as power series in the surface charge density or surface potential of the particles were obtained, and these results demonstrate that the effect of the double-layer overlap is quite significant even for the case of thin double layers.

Recently, Ohshima [31] derived general expressions for the sedimentation velocity and potential for a concentrated suspension of identical soft spherical particles on the basis of the Kuwabara cell model for the case of low electrostatic potentials and nonoverlapping double layers of adjacent particles, neglecting the polarization (relaxation) effect of each double layer. In this article, the unit cell model is used to study the sedimentation phenomena in a suspension of identical charged porous spheres. The overlap of adjacent double layers is allowed and the polarization effect in the diffuse layer surrounding each particle is included. No assumption is made about the thickness of the double layer relative to the dimension of the particle. Both the Happel model and the Kuwabara model are considered. The basic electrokinetic equations are linearized assuming that the electrolyte ion concentrations, the electrostatic potential, and the fluid pressure have only a slight deviation from equilibrium due to the motion of the particle. Through the use of a regular perturbation method with the fixed charge density of the particle as the small perturbation parameter, the ion concentration (or electrochemical potential), electric potential, and fluid velocity profiles are determined by solving these linearized electrokinetic equations subject to the appropriate boundary conditions. Analytical expressions for the settling velocity of the charged porous spheres in the solution of a symmetrically charged electrolyte and for the sedimentation potential in the suspension are obtained in closed forms.

\section{Basic electrokinetic equations}

We consider the sedimentation (or any other body-forcedriven motion) of a statistically homogeneous distribution of identical charged porous spherical particles in a bounded liquid solution containing $M$ ionic species at the steady state. The acceleration of gravity (or the uniformly imposed body force field) equals $g \mathbf{e}_{z}$ and the sedimentation (or migration) velocity of the porous particles is $U \mathbf{e}_{z}$, where $\mathbf{e}_{z}$ is the unit vector in the positive $z$-direction. As shown in Fig. 1, we employ a unit cell model in which each particle of radius $a$ is surrounded by a concentric spherical shell of suspending solution having an outer radius of $b$ such that the particle/cell volume ratio is equal to the apparent particle volume fraction $\varphi$ throughout the entire suspension; viz., $\varphi=(a / b)^{3}$. The cell as a whole is electrically neutral. The origin of the spherical coordinate system $(r, \theta, \phi)$ is taken at the center of the particle and the axis $\theta=0$ points toward the positive $z$-direction. Obviously, the problem for each cell is axially symmetric about the $z$-axis.

It is assumed that the magnitude of the particle velocity is not large and hence that the electric double layer surrounding the particle is only slightly distorted from the equilibrium state, where the particle and fluid are at rest. Therefore, the concen- 


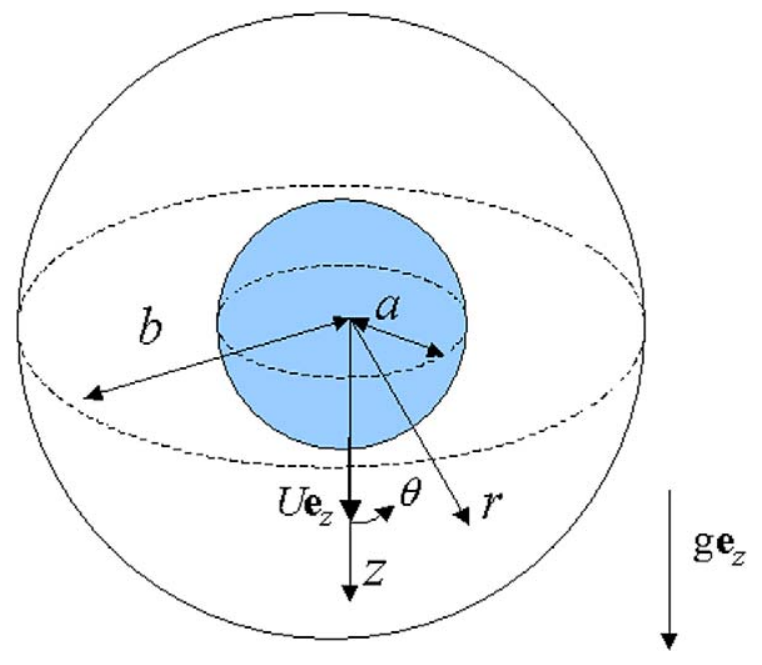

Fig. 1. Geometrical sketch for the sedimentation of a charged porous sphere at the center of a spherical cell.

tration (number density) distribution $n_{m}(r, \theta)$ of species $m$, the electric potential distribution $\psi(r, \theta)$, and the pressure distribution $p(r, \theta)$ can be expressed as

$n_{m}=n_{m}^{(\mathrm{eq})}+\delta n_{m}$,
$\psi=\psi^{(\mathrm{eq})}+\delta \psi$,
$p=p^{(\mathrm{eq})}+\delta p$,

where $n_{m}^{(\mathrm{eq})}(r), \psi^{(\mathrm{eq})}(r)$, and $p^{(\mathrm{eq})}(r, \theta)$ are the equilibrium distributions of the concentration of species $m$, electric potential, and pressure, respectively, and $\delta n_{m}(r, \theta), \delta \psi(r, \theta)$, and $\delta p(r, \theta)$ are the corresponding small deviations from the equilibrium state. The equilibrium concentration of each ionic species is related to the equilibrium potential by the Boltzmann distribution.

It can be shown that the small perturbed quantities $\delta n_{m}, \delta \psi$, and $\delta p$, together with the fluid velocity field $\mathbf{u}(r, \theta)$, satisfy the following set of linearized electrokinetic equations $[18,30]$ :

$\nabla \cdot \mathbf{u}=0$

$\nabla^{2} \mathbf{u}-h(r) \lambda^{2} \mathbf{u}=\frac{1}{\eta} \nabla \delta p-\frac{\varepsilon}{4 \pi \eta}\left[\nabla^{2} \psi^{(\mathrm{eq})} \nabla \delta \psi\right.$

$$
\left.+\nabla^{2} \delta \psi \nabla \psi(\mathrm{eq})\right]-\frac{1}{\eta} h(r) Q \nabla \delta \psi
$$

$\nabla^{2} \delta \mu_{m}=\frac{z_{m} e}{k T}\left[\nabla \psi^{(\mathrm{eq})} \cdot \nabla \delta \mu_{m}-\frac{k T}{D_{m}} \nabla \psi^{(\mathrm{eq})} \cdot \mathbf{u}\right]$,

$$
m=1,2, \ldots, M \text {, }
$$

$$
\begin{aligned}
\nabla^{2} \delta \psi= & -\frac{4 \pi}{\varepsilon} \sum_{m=1}^{M} \frac{z_{m} e n_{m}^{\infty}}{k T} \exp \left[-\frac{z_{m} e \psi(\mathrm{eq})}{k T}\right] \\
& \times\left(\delta \mu_{m}-z_{m} e \delta \psi\right) .
\end{aligned}
$$

Here, $\delta \mu_{m}(r, \theta)$ is defined as a linear combination of $\delta n_{m}$ and $\delta \psi$ on the basis of the concept of the electrochemical potential energy [10],

$\delta \mu_{m}=\frac{k T}{n_{m}^{(\mathrm{eq})}} \delta n_{m}+z_{m} e \delta \psi$ $n_{m}^{\infty}$ is the concentration of the type $m$ ions in the bulk (electrically neutral) solution where the equilibrium potential is set equal to zero; $\eta$ is the viscosity of the fluid (the available evidence [32] suggests that it is reasonable to assume the same value of $\eta$ inside and outside the porous particle); $\lambda=(f / \eta)^{1 / 2}$, where $f$ is the hydrodynamic friction coefficient inside the porous particle per unit volume of the fluid (which accounts for the hindrance to the convective transport of the electrolyte solution caused by the frictional segments); $h(r)$ is a unit step function which equals unity if $r \leqslant a$, and zero otherwise; $Q$ is the fixed charge density inside the porous particle; $D_{m}$ and $z_{m}$ are the diffusion coefficient and valence, respectively, of species $m ; e$ is the elementary electric charge; $k$ is Boltzmann's constant; $T$ is the absolute temperature; and $\varepsilon=4 \pi \varepsilon_{0} \varepsilon_{\mathrm{r}}$, where $\varepsilon_{\mathrm{r}}$ is the relative permittivity of the electrolyte solution and $\varepsilon_{0}$ is the permittivity of a vacuum. We assume that the values of $\eta$, $f, \varepsilon$, and $D_{m}$ are constant.

Note that $f$ can be expressed as $6 \pi \eta a_{\mathrm{S}} N_{\mathrm{S}}$ in the freedraining limit, where $N_{\mathrm{S}}$ and $a_{\mathrm{S}}$ are the number density and the Stokes radius, respectively, of the hydrodynamic frictional segments of the porous particle, and the reciprocal of the parameter $\lambda$ is the shielding length characterizing the extent of flow penetration inside the porous particle. For some model porous particles made of steel wool (in glycerin-water solution) [33] and plastic foam slab (in silicon oil) [34], experimental values of $1 / \lambda$ can be as high as $0.4 \mathrm{~mm}$, whereas in the surface regions of human erythrocytes [35], rat lymphocytes [36], and grafted polymer microcapsules [37] in salt solutions, values of $1 / \lambda$ were found to be about $3 \mathrm{~nm}$. Note that $1 / \lambda^{2}$ is the socalled "permeability" of the porous medium, which is related to its pore size and porosity and characterizes the dynamic behavior of the viscous fluid in it.

The conditions to be satisfied inside the porous particle are

$\mathbf{u}, \delta \mu_{m}$, and $\delta \psi$ are finite.

The boundary conditions at the particle surface $\mathrm{S}$ (at $r=a$ ) are

$\left.\mathbf{u}\right|_{\mathrm{S}^{+}}=\left.\mathbf{u}\right|_{\mathrm{S}^{-}}$,

$\left.\mathbf{n} \cdot \boldsymbol{\sigma}\right|_{\mathrm{S}^{+}}=\left.\mathbf{n} \cdot \boldsymbol{\sigma}\right|_{\mathrm{S}^{-}}$,

$\left.\delta \mu_{m}\right|_{\mathrm{S}^{+}}=\left.\delta \mu_{m}\right|_{\mathrm{S}^{-}}$,

$\left.\nabla \delta \mu_{m}\right|_{\mathrm{S}^{+}}=\left.\nabla \delta \mu_{m}\right|_{\mathrm{S}^{-}}$,

$\left.\delta \psi\right|_{\mathrm{S}^{+}}=\left.\delta \psi\right|_{\mathrm{S}^{-}}$,

$\left.\nabla \delta \psi\right|_{\mathrm{S}^{+}}=\left.\nabla \delta \psi\right|_{\mathrm{S}^{-}}$,

where the superscripts + and - to $S$ represent the external and internal sides, respectively, to the surface of the particle, $\mathbf{n}$ is the unit vector outwardly normal to the particle surface, and $\sigma$ is the hydrodynamic stress of the fluid. Equations (8a) and (8b) are the continuity requirements of the fluid velocity and stress at the particle surface which are physically realistic and mathematically consistent boundary conditions for the present problem $[32,38,39]$. Since we take the same fluid viscosity inside and outside the porous particle and use the fluid velocity continuity given by Eq. (8a), Eq. (8b) is equivalent to the continuity of pressure. Equations $(8 \mathrm{c})$ and $(8 \mathrm{~d})$ state that the concentrations 
and fluxes of the ionic species must be continuous at the particle surface, while Eqs. (8e) and (8f) indicate that the electrostatic potential and electric field are also continuous. The continuity of the electric field results from the assumption that the relative permittivity of the solution takes the same value both inside and outside the porous particle. In the present system, the total fluid stress, which consists of the hydrodynamic stress and the Maxwell stress, is continuous at the particle surface. The boundary condition for the continuity of hydrodynamic stress given by Eq. (8b) comes from the fact of continuous Maxwell stress that can be deduced from Eqs. (1b) and (8f) [17].

The boundary conditions at the virtual surface of the cell, in which the overlap of the electric double layers of adjacent particles is allowed, are

$$
\begin{gathered}
r=b: \quad u_{r}=-U \cos \theta, \\
\tau_{r \theta}=\eta\left[r \frac{\partial}{\partial r}\left(\frac{u_{\theta}}{r}\right)+\frac{1}{r} \frac{\partial}{\partial \theta}\right. \\
(\text { for Happel model }), \\
(\nabla \cdot \mathbf{u})_{\phi}=\frac{1}{r} \frac{\partial}{\partial r}\left(r u_{\theta}\right)-\frac{1}{r} \\
(\text { for Kuwabara model }), \\
\frac{\partial \delta \mu_{m}}{\partial r}=0, \\
\frac{\partial \delta \psi}{\partial r}=0,
\end{gathered}
$$$$
\tau_{r \theta}=\eta\left[r \frac{\partial}{\partial r}\left(\frac{u_{\theta}}{r}\right)+\frac{1}{r} \frac{\partial u_{r}}{\partial \theta}\right]=0
$$$$
(\nabla \cdot \mathbf{u})_{\phi}=\frac{1}{r} \frac{\partial}{\partial r}\left(r u_{\theta}\right)-\frac{1}{r} \frac{\partial u_{r}}{\partial \theta}=0
$$

where $u_{r}$ and $u_{\theta}$ are the $r$ and $\theta$ components, respectively, of the fluid velocity $\mathbf{u}$. Note that the Happel cell model [23] assumes that the radial velocity and the shear stress of the fluid on the outer boundary of the cell are zero, while the Kuwabara cell model [24] assumes that the radial velocity and the vorticity of the fluid are zero there. Equation (9a) takes a reference frame that the particle is at rest and the velocity of the fluid at the outer boundary of the cell is the particle velocity in the opposite direction. The conditions (9a), (9d), and (9e) imply that there are no net flows of fluid, ionic species, and electric current between adjacent cells. They are valid because the suspension of the particles is bounded by impermeable, inert, and nonconductive walls. Thus, the effect of the backflow of fluid occurring in a container is included in both cell models.

For the sedimentation of a suspension of uncharged spherical particles, both the Happel and the Kuwabara models give qualitatively the same flow fields and approximately comparable drag forces on the particle in a cell. However, the Happel model has a significant advantage in that it does not require an exchange of mechanical energy between the cell and the environment [40].

\section{Solution of the electrokinetic equations for symmetric electrolytes}

We now consider the sedimentation of a charged porous sphere in a unit cell filled with the solution of a symmetrically charged binary electrolyte with a constant bulk concentration $n^{\infty}\left(M=2, z_{+}=-z_{-}=Z, n_{+}^{\infty}=n_{-}^{\infty}=n^{\infty}\right.$, where subscripts + and - refer to the cation and anion, respectively). We first seek the solution of $\psi^{(\mathrm{eq})}$ which appears in Eqs. (3)-(5) and is governed by the equilibrium Poisson-Boltzmann equation,

$\nabla^{2} \psi^{(\mathrm{eq})}=\frac{k T}{Z e} \kappa^{2} \sinh \left[\frac{Z e \psi^{(\mathrm{eq})}}{k T}\right]-h(r) \frac{4 \pi Q}{\varepsilon}$,

where $\kappa=\left(8 \pi Z^{2} e^{2} n^{\infty} / \varepsilon k T\right)^{1 / 2}$ is the Debye screening parameter. The boundary conditions for $\psi^{(\mathrm{eq})}$ are

$r=0: \quad \psi^{(\mathrm{eq})}$ is finite,

$r=a: \quad \psi^{(\mathrm{eq})}$ and $\frac{\mathrm{d} \psi^{(\mathrm{eq})}}{\mathrm{d} r}$ are continuous,

$r=b: \quad \frac{\mathrm{d} \psi^{(\mathrm{eq})}}{\mathrm{d} r}=0$.

The solution to Eqs. (10)-(13) is

$\psi^{(\mathrm{eq})}(r)=\psi_{\mathrm{eq} 1}(r) \bar{Q}+\mathrm{O}\left(\bar{Q}^{3}\right)$,

where

$$
\begin{aligned}
\psi_{\mathrm{eq} 1}= & \frac{k T}{Z e}\left\{1-\left[(1-\kappa a) e^{\kappa a}+\frac{\alpha(\kappa a)}{\alpha(\kappa b)}\right.\right. \\
& \left.\left.\times(\kappa b-1) e^{\kappa b}\right] \frac{\sinh (\kappa r)}{\kappa r}\right\} \quad \text { if } 0 \leqslant r \leqslant a, \\
\psi_{\text {eq } 1}= & \frac{k T}{Z e} \frac{\alpha(\kappa a)}{\alpha(\kappa b)} \frac{1}{\kappa r}[\kappa b \cosh (\kappa b-\kappa r)-\sinh (\kappa b-\kappa r)] \\
\text { if } a \leqslant & r \leqslant b,
\end{aligned}
$$

the function $\alpha(x)$ is defined by Eq. (A.3a) in Appendix A, and $\bar{Q}=4 \pi Z e Q / \varepsilon \kappa^{2} k T$ is the nondimensional charge density of the porous particle. Expression (14) for $\psi^{(\mathrm{eq})}$ as a power series in $\bar{Q}$ up to $\mathrm{O}(\bar{Q})$ is the equilibrium solution for the linearized Eq. (10) that is valid for small values of the electric potential (the Debye-Huckel approximation). That is, the fixed charge density $Q$ of the particle must be small enough for the potential to remain small. Note that $\psi^{(\mathrm{eq})}$ and $n_{ \pm}^{(\mathrm{eq})}$ depend on $r$ only due to spherical symmetry, and the $\mathrm{O}\left(\bar{Q}^{2}\right)$ term in Eq. (14) for $\psi^{(\text {eq })}$ disappears only for the case of symmetric electrolytes.

To solve the small quantities $\mathbf{u}, \delta p, \delta \mu_{ \pm}$, and $\delta \psi$ in terms of the particle velocity $U$ when the parameter $\bar{Q}$ is small, these variables can be written as regular perturbation expansions in powers of $\bar{Q}$,

$\mathbf{u}=\mathbf{u}_{0}+\mathbf{u}_{1} \bar{Q}+\mathbf{u}_{2} \bar{Q}^{2}+\cdots$

$\delta p=p_{0}+p_{1} \bar{Q}+p_{2} \bar{Q}^{2}+\cdots$,

$\delta \mu_{ \pm}=\mu_{1 \pm} \bar{Q}+\mu_{2 \pm} \bar{Q}^{2}+\cdots$,

$\delta \psi=\psi_{1} \bar{Q}+\psi_{2} \bar{Q}^{2}+\cdots$,

$U=U_{0}+U_{1} \bar{Q}+U_{2} \bar{Q}^{2}+\cdots$,

where the functions $\mathbf{u}_{i}, p_{i}, \mu_{i \pm}, \psi_{i}$, and $U_{i}$ are independent of $\bar{Q}$. The zeroth-order terms of both $\delta \mu_{ \pm}$and $\delta \psi$ disappear due to not imposing a macroscopic electrolyte gradient and electric field.

Substituting the expansions given by Eq. (16) and $\psi^{(\mathrm{eq})}$ given by Eq. (14) into the governing equations (2)-(5) and boundary conditions (7)-(9), and equating like powers of $\bar{Q}$ on both sides of the respective equations, we can obtain a set of linear differential equations and boundary conditions for each set 
of the functions $\mathbf{u}_{i}, p_{i}, \mu_{i \pm}$, and $\psi_{i}$ with $i$ equal to 0,1 , and 2 . After solving these perturbation equations, the results for the $r$ and $\theta$ components of $\mathbf{u}$ (to the order $\bar{Q}^{2}$ ), $\delta \mu_{ \pm}$, and $\delta \psi$ (to the order $\bar{Q}$, which will be sufficient for the calculation of the sedimentation velocity and sedimentation potential to the order $\bar{Q}^{2}$ ) can be written as

$$
\begin{aligned}
u_{r}= & \left\{U_{0} F_{0}(r)-U_{1} F_{0}(r) \bar{Q}+\left[U_{0} F_{2}(r)+U_{2} F_{0}(r)\right] \bar{Q}^{2}\right\} \\
& \times \cos \theta+O\left(\bar{Q}^{3}\right), \\
u_{\theta}= & -\frac{1}{2 r} \frac{\mathrm{d}}{\mathrm{d} r} r^{2}\left\{U_{0} F_{0}(r)-U_{1} F_{0}(r) \bar{Q}\right. \\
& \left.+\left[U_{0} F_{2}(r)+U_{2} F_{0}(r)\right] \bar{Q}^{2}\right\} \sin \theta+O\left(\bar{Q}^{3}\right),
\end{aligned}
$$

$\delta \mu_{ \pm}=U_{0} F_{ \pm}(r) \cos \theta \bar{Q}+O\left(\bar{Q}^{2}\right)$,

$\delta \psi=U_{0} F_{\psi}(r) \cos \theta \bar{Q}+O\left(\bar{Q}^{2}\right)$.

Here, the functions $F_{i}(r)$ (with $i$ equal to 0 and 2), $F_{ \pm}(r)$, and $F_{\psi}(r)$ for both the Happel and the Kuwabara cell models are defined by Eqs. (A.1), (A.8), and (A.9) in Appendix A. Since $F_{ \pm}(r)$ and $F_{\psi}(r)$ are influenced by the fluid flow via $F_{0}(r)$, the leading order of the effect of the relaxation (or polarization) of the diffuse ions in the electric double layer surrounding the particle is included in the solution for $\delta \mu_{ \pm}$and $\delta \psi$ up to the order $\bar{Q}$.

\section{Sedimentation velocity}

The total force exerted on the charged porous sphere settling in the electrolyte solution within a unit cell can be expressed as the sum of the gravitational force (and buoyant force), the electrostatic force, and the hydrodynamic force acting on the particle. The gravitational force is given by

$\mathbf{F}_{\mathrm{g}}=\frac{4}{3} \pi a^{3}\left(1-\varepsilon_{\mathrm{p}}\right)\left(\rho_{\mathrm{p}}-\rho\right) g \mathbf{e}_{z}$,

where $\varepsilon_{\mathrm{p}}$ and $\rho_{\mathrm{p}}$ are the porosity and true mass density of the porous particle, respectively, $\rho$ is the mass density of the fluid, and $g \mathbf{e}_{z}$ is the gravitational acceleration.

The electric force acting on the porous sphere is defined by

$\mathbf{F}_{\mathrm{e}}=-\int_{r \leqslant a} Q \nabla \psi \mathrm{d} \mathbf{x}$

Substituting Eqs. (1b) and (19) into Eq. (21), and using the fact that the net electric force acting on the particle at the equilibrium state is zero, one has

$\mathbf{F}_{\mathrm{e}}=-\frac{\varepsilon}{3} \frac{k T}{Z e}(\kappa a)^{2} U_{0} F_{\psi}(a) \bar{Q}^{2} \mathbf{e}_{z}$

The hydrodynamic drag force acting on the porous sphere is given by

$\mathbf{F}_{\mathrm{h}}=\int_{r \leqslant a} f \mathbf{u}(\mathbf{x}) \mathrm{d} \mathbf{x}$.

Substitution of Eq. (17) into the above equation results in

$\mathbf{F}_{\mathrm{h}}=\frac{4 \pi}{3} \eta \lambda^{2} a^{3}\left\{U_{0} F_{0}(a)+U_{1} F_{0}(a) \bar{Q}\right.$

$$
\left.+\left[U_{0} F_{2}(a)+U_{2} F_{0}(a)\right] \bar{Q}^{2}\right\} \mathbf{e}_{z} .
$$

At the steady state, the total force acting on the settling particle (or the unit cell) is zero. Applying this constraint to the summation of Eqs. (20), (22), and (24) for a symmetric electrolyte, we obtain the sedimentation velocity of the charged porous sphere in the expansion form of Eq. (16e) with the first three coefficients as

$U_{0}=\frac{-\left(1-\varepsilon_{\mathrm{p}}\right)\left(\rho_{\mathrm{p}}-\rho\right) g}{\eta \lambda^{2}\left[C_{01}+\alpha(\lambda a) C_{02}\right]}$,

$U_{1}=0$,

$U_{2}=-U_{0}\left[\frac{F_{2}(a)}{F_{0}(a)}-\left(\frac{\varepsilon \kappa^{2} k T}{4 \pi \eta \lambda^{2} a z e}\right) \frac{F_{\psi}(a)}{F_{0}(a)}\right]$,

where the coefficients $C_{01}$ and $C_{02}$ are given by Eq. (A.4) for the Happel cell model and by Eq. (A.5) for the Kuwabara cell model in Appendix A. $U_{0}$ is the settling velocity of an uncharged porous sphere in the cell [41]. The definite integrals in the functions $F_{2}(a)$ and $F_{\psi}(a)$ in Eq. (25c) defined by Eqs. (A.1) and (A.9) can be calculated numerically. Note that the correction for the effect of the fixed charge density to the particle velocity starts from the second order $Q^{2}$, instead of the first order $Q$. The reason is that this effect is due to the interaction between the particle charges and the local induced sedimentation potential gradient; both are of order $Q$ and thus the correction is of order $Q^{2}$.

Substitution of Eq. (25) into Eq. (16e) results in an expression for the sedimentation velocity as a perturbation expansion in powers of $Q$,

$U=U_{0}\left[1-(\kappa a)^{4} H \bar{Q}^{2}+O\left(\bar{Q}^{3}\right)\right]$.

Here, the dimensionless coefficient $H$ is a function of the parameters $\kappa a, \lambda a$, and $\varphi$ for a given electrolyte solution,

$H=-\frac{U_{2}}{(\kappa a)^{4} U_{0}}$.

The numerical result of $H$ calculated by using Eqs. (25a) and $(25 \mathrm{c})$ will be presented in Section 6 . Note that $(\kappa a)^{2} \bar{Q}$ $\left(=4 \pi a^{2} Z e Q / \varepsilon k T\right)$ is independent of $\kappa$ or $n^{\infty}$ for a constant fixed charge density $Q$.

\section{Sedimentation potential}

The electric fields around the individual charged particles undergoing sedimentation in a suspension superimpose to result in a sedimentation potential gradient. For a homogeneous suspension of identical spherical particles, the sedimentation potential field is uniform and can be regarded as the average of the gradient of electric potential over a sufficiently large volume of the suspension to contain many particles. In order to calculate this field, the requirement that there exists no net electric current in the suspension must be satisfied. For identical charged porous spheres suspended in a symmetric electrolyte with the absolute value of valence $Z$, the sedimentation potential field obtained from the unit cell model can be expressed as [18]

$\mathbf{E}_{\mathrm{SED}}=\frac{4 \pi b^{2} Z^{2} e^{2} n^{\infty} N}{3 k T V \Lambda^{\infty}} U_{0}\left\{\left[D_{+}\left(r \frac{\mathrm{d} F_{+}}{\mathrm{d} r}-F_{+}\right)\right.\right.$ 


$$
\left.\left.-D_{-}\left(r \frac{\mathrm{d} F_{-}}{\mathrm{d} r}-F_{-}\right)\right]_{r=b} \bar{Q}+O\left(\bar{Q}^{3}\right)\right\} \mathbf{e}_{z} .
$$

Substituting Eq. (25a) for $U_{0}$ and Eq. (A.8) for $F_{ \pm}(r)$ after the numerical integration into the above equation, we obtain this field as

$\mathbf{E}_{\mathrm{SED}}=-\left(1-\varepsilon_{\mathrm{p}}\right)\left(\rho_{\mathrm{p}}-\rho\right) \frac{\varphi g}{\Lambda^{\infty}} \mu_{\mathrm{E}} \mathbf{e}_{z}$,

where

$$
\begin{aligned}
\mu_{\mathrm{E}}= & \frac{Q}{\eta \lambda^{2} \alpha(\kappa b)\left[C_{01}+\alpha(\lambda a) C_{02}\right]}\left\{6 L_{1}(\lambda a)^{3}\left(\varphi^{-1 / 3}-\varphi^{-2}\right)\right. \\
& \times\left[\frac{\lambda a \sinh (\lambda a) \cosh (\kappa a)-\kappa a \cosh (\lambda a) \sinh (\kappa a)}{(\lambda a)^{2}-(\kappa a)^{2}}\right. \\
& \left.-\frac{\sinh (\lambda a) \sinh (\kappa a)}{(\lambda a)(\kappa a)}\right] \\
& +\frac{3 L_{1} \varphi^{-2}}{(\kappa a)^{3}}\left[2 A_{2} \varphi^{5 / 3}+(\lambda a)^{2}\left(1+2 \varphi^{5 / 3}\right) \alpha(\lambda a)\right] \\
& \times\left[3 \alpha(\kappa a)-(\kappa a)^{2} \sinh (\kappa a)\right] \\
& +\frac{\alpha(\kappa a)}{(\kappa a)^{2}}\left[(\lambda a \kappa a)^{2} B_{1} \varphi^{-8 / 3}+10(\lambda a)^{2} A_{4} \varphi^{-4 / 3}\right. \\
& \left.-2(\lambda a)^{2} B_{1} \varphi^{-2}-(\kappa a)^{2} B_{2} \varphi^{-3}\right] \\
& -\frac{\alpha(\kappa a) L_{2}}{(\kappa a)^{5}\left[(\lambda a)^{2}\left(\kappa^{4} a^{4}+15 \kappa^{2} a^{2}+30\right) A_{4} \varphi^{-1 / 3}\right.} \\
& \left.-(\kappa a)^{2}\left[(\lambda a \kappa a)^{2}\left(A_{4}-B_{1}\right)+\left(\kappa^{2} a^{2}+3\right) B_{2}\right] \varphi^{-2}\right] \\
& +\frac{\alpha(\kappa a) L_{3}}{(\kappa a)^{4}}\left[5(\lambda a)^{2}\left(\kappa^{2} a^{2}+6\right) A_{4} \varphi^{-2 / 3}\right. \\
& \left.\left.+(\kappa a)^{2}\left[2(\lambda a)^{2} B_{1}-3 B_{2}\right] \varphi^{-7 / 3}\right]\right\}+O\left(\bar{Q}^{3}\right)
\end{aligned}
$$

for the Happel model, and

$$
\begin{aligned}
\mu_{\mathrm{E}}= & \frac{Q \varphi^{2}}{45 \eta \lambda^{2} \alpha(\kappa b) \alpha(\lambda a)}\left\{30 \lambda a L_{1}\left(\varphi^{-1}-\varphi^{-2}\right)\right. \\
& \times\left[\frac{\lambda a \sinh (\lambda a) \cosh (\kappa a)-\kappa a \cosh (\lambda a) \sinh (\kappa a)}{(\kappa a)^{2}-(\lambda a)^{2}}\right. \\
& \left.-\frac{\sinh (\lambda a) \cosh (\kappa a)}{(\lambda a)(\kappa a)}\right]+\frac{15 L_{1}\left(2 \varphi^{-1}+\varphi^{-2}\right) \alpha(\lambda a)}{(\kappa a)^{3}} \\
& \times\left[3 \alpha(\kappa a)-(\kappa a)^{2} \sinh (\kappa a)\right] \\
& +\alpha(\kappa a)\left[\left(2 A_{2} \varphi-5 A_{4}\right) \varphi^{-2}+18(\lambda a)^{2} \varphi^{-8 / 3}\right. \\
& \left.-5\left(A_{1}+A_{4} \varphi\right) \varphi^{-3}\right]-\frac{\alpha(\kappa a) L_{2}}{(\kappa a)^{5}\left[3 ( \lambda a ) ^ { 2 } \left(\kappa^{4} a^{4}\right.\right.} \\
& \left.+15 \kappa^{2} a^{2}+30\right) \alpha(\lambda a) \varphi^{-1}-(\kappa a)^{2}\left[5\left(\kappa^{2} a^{2}+3\right)\right. \\
& \times\left(A_{1}+A_{4} \varphi\right)-(\kappa a)^{2}\left(2 A_{2} \varphi-5 A_{4}\right) \\
& \left.\left.-15(\lambda a \kappa a)^{2} \alpha(\lambda a)\right] \varphi^{-2}\right] \\
& +\frac{15 \alpha(\kappa a) L_{3}}{(\kappa a)^{4}}\left[(\lambda a)^{2}\left(\kappa^{2} a^{2}+6\right) \alpha(\lambda a) \varphi^{-4 / 3}+(\kappa a)^{2}\right. \\
& \left.\left.\times\left[2(\lambda a)^{2} \alpha(\lambda a)-A_{1}-A_{4} \varphi\right] \varphi^{-7 / 3}\right]\right\}+O\left(\bar{Q}^{3}\right)
\end{aligned}
$$

for the Kuwabara model. In the above equations, $A_{i}, L_{i}$, and $B_{i}$ are defined by Eqs. (A.7), (A.10), and (A.11), and $\varphi=(a / b)^{3}$ is the apparent volume fraction of the particles in the suspension. Equation (29) is an Onsager reciprocal relation connecting the sedimentation potential with the electrophoretic mobility derived by de Groot et al. [9] on the basis of irreversible thermodynamics. Evidently, the electrophoretic mobility $\mu_{\mathrm{E}}$ is independent of the ionic diffusion coefficients and is a function of the parameters $\kappa a, \lambda a$, and $\varphi$ only.

\section{Results and discussion}

For the limiting case of an infinitely dilute suspension of porous spheres $(\varphi=0)$, the quantities $U_{0}$ and $\mu_{\mathrm{E}}$ in expressions (25a) and (30) for the sedimentation velocity and potential reduce to

$$
\begin{aligned}
U_{0}= & \left(1-\varepsilon_{\mathrm{p}}\right)\left(\rho_{\mathrm{p}}-\rho\right) \frac{2 a^{2} g}{9 \eta}\left[2(\lambda a) A_{1} \alpha(\lambda a)\right]^{-1}, \\
\mu_{\mathrm{E}}= & \frac{Q}{\eta \lambda^{2}}\left\{1+\frac{1}{3}\left(\frac{\lambda}{\kappa}\right)^{2}\left(1+e^{-2 \kappa a}-\frac{1-e^{-2 \kappa a}}{\kappa a}\right)\right. \\
& +\frac{1}{3}\left(\frac{\lambda^{2}}{\lambda^{2}-\kappa^{2}}\right)\left(1+\frac{1}{\kappa a}\right) \\
& \left.\times\left[\left(\frac{\lambda}{\kappa}\right)^{2} \frac{\kappa a\left(1+e^{-2 \kappa a}\right)-1+e^{-2 \kappa a}}{\lambda a \operatorname{coth}(\lambda a)-1}-1+e^{-2 \kappa a}\right]\right\} \\
& +O\left(\bar{Q}^{2}\right) .
\end{aligned}
$$

These reduced results are the same as the formulas for $U_{0}$ and $\mu_{\mathrm{E}}$ obtained previously $[11,14,18]$ for a single porous sphere in an unbounded electrolyte.

According to Eqs. (25)-(27), the sedimentation velocity of charged porous spheres in a given electrolyte solution can be calculated to the order of $Q^{2}$. The numerical results of the dimensionless coefficient $H$ for particles in the aqueous solution of $\mathrm{HCl}$ at room temperature as a function of the parameters $\kappa a, \lambda a$, and $\varphi$ are plotted in Figs. 2-4. The value $\varepsilon k^{2} T^{2} / 4 \pi \eta D z^{2} e^{2}=0.259$ with $D_{+}=D_{-}=D$ [42] is used in the calculations, and the results are presented up to $\varphi=0.74$, which corresponds to the maximum attainable volume fraction for a swarm of identical spheres [43]. The fact that $H$ is always positive demonstrates that the presence of the particle charges reduces the sedimentation rate for any volume fraction of particles in the suspension. This retardation on the settling of the charged particles reflects the electrophoretic effect on the particles in the direction opposite to gravity caused by the sedimentation potential gradient induced in the suspension.

For fixed values of $\lambda a$ and $\varphi$, the coefficient $H$ has a maximum at some finite value of $\kappa a$ and vanishes in the limits $\kappa a \rightarrow 0$ and $\kappa a \rightarrow \infty$. The reason for this behavior is obvious. The limit $\kappa a \rightarrow 0$ means that the presence of the counterions around each particle is negligible and the perturbation quantities $\delta n_{m}$ and $\delta \psi$ disappear, while the limit $\kappa a \rightarrow \infty$ indicates that the total charge density is zero everywhere and the total electric force on the particle vanishes. Note that the location of this maximum shifts to greater $\kappa a$ as $\varphi$ increases, keeping the value of $\lambda a$ unchanged, but is not a sensitive function of $\lambda a$, as shown in Fig. 2. For given values of $\kappa a$ and $\varphi$, the coefficient $H$ increases monotonically with decreasing $\lambda a$. In the 


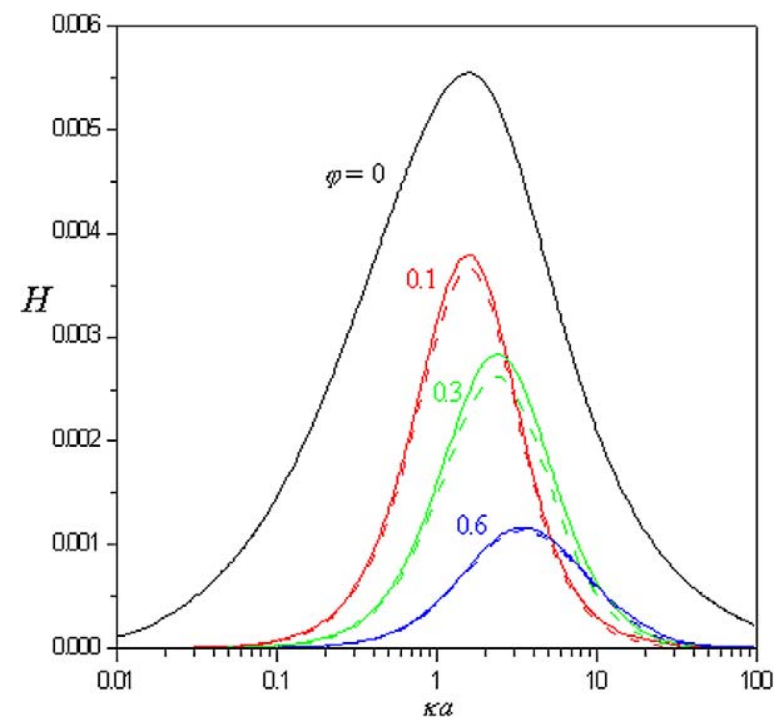

(a)

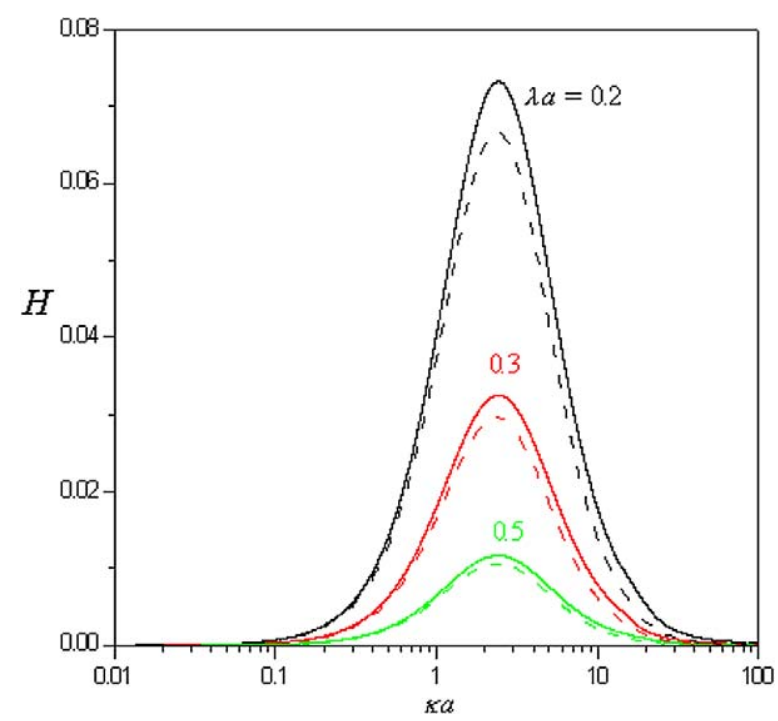

(b)

Fig. 2. Plots of the dimensionless coefficient $H$ in Eq. (26) for settling charged porous spheres in the $\mathrm{KCl}$ solution versus the parameter $\kappa a$ : (a) $\lambda a=1$; (b) $\varphi=0.3$. The solid and dashed curves represent the calculations for the Happel and Kuwabara cell models, respectively.

limit $\lambda a \rightarrow \infty$, the porous particles behave like impermeable conductive spheres and thus $H=0$ (the sedimentation velocity of the particle limits to the Stokes velocity). At small values of $\lambda a$ (say, less than 0.05), the porous particles are nearly free-drained, and their velocity might be large that the perturbation quantities become comparable in magnitude with the equilibrium values. Therefore, our perturbation result will not be adaptable for this case owing to disobeying the assumption that the system is only slightly distorted from equilibrium. For specified values of $\kappa a$ and $\lambda a$ in a broad range, $H$ is not a monotonic function of $\varphi$ and has a maximal value. The location of this maximum shifts to greater $\varphi$ as $\kappa a$ increases but is not a sensitive function of $\lambda a$.

Figs. 2-4 illustrate that, for any combination of $\kappa a, \lambda a$, and $\varphi$, the Kuwabara model predicts a smaller value for the coeffi-

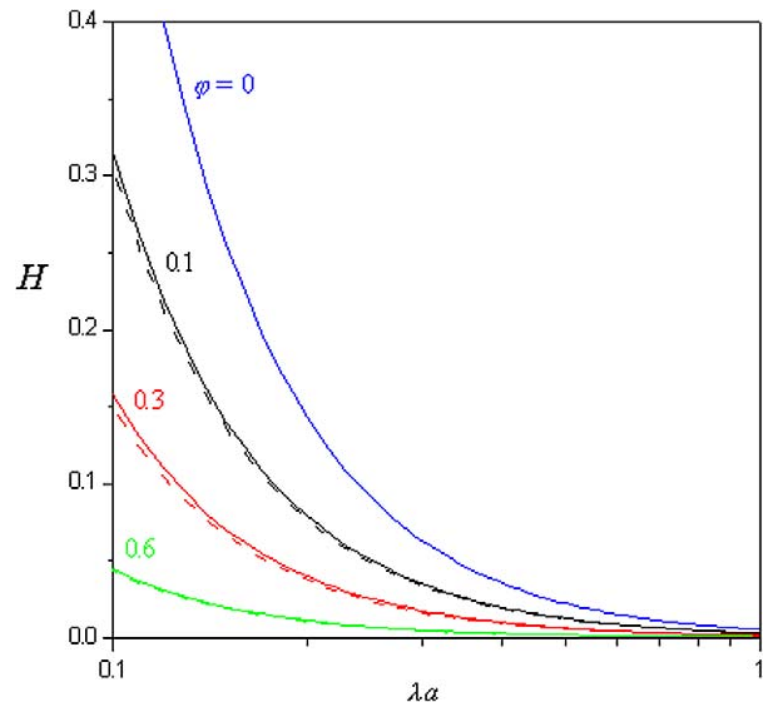

(a)

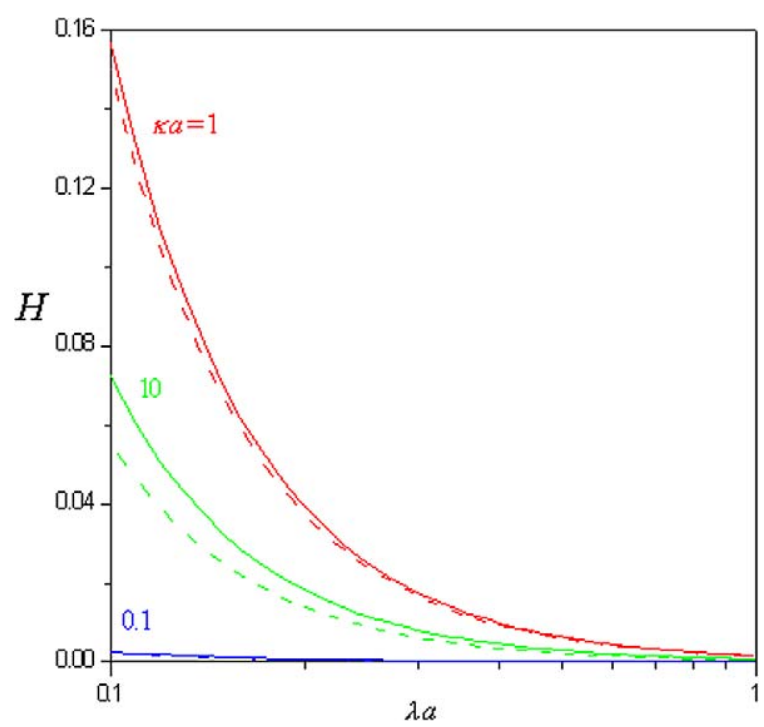

(b)

Fig. 3. Plots of the dimensionless coefficient $H$ in Eq. (26) for settling charged porous spheres in the $\mathrm{KCl}$ solution versus the parameter $\lambda a$ : (a) $\kappa a=1$; (b) $\varphi=0.3$. The solid and dashed curves represent the calculations for the Happel and Kuwabara cell models, respectively.

cient $H$ (or a weaker particle concentration dependence for the sedimentation of the suspension) than the Happel model does. This occurs because the zero-vorticity model yields a larger energy dissipation in the cell than that due to particle drag alone, owing to the additional work done by the stresses at the outer boundary [40]. In general, the qualitative and quantitative differences in $H$ between the two models are not significant.

In Figs. 5-7, the numerical results for the dimensionless sedimentation potential or electrophoretic mobility $\eta \lambda^{2} \mu_{\mathrm{E}} / Q$ in a suspension of identical charged porous spheres calculated from Eq. (30) are plotted as a function of the parameters $\kappa a, \lambda a$, and $\varphi$. As expected, $\eta \lambda^{2} \mu_{\mathrm{E}} / Q$ is always a positive value. For a given value of $\lambda a, \eta \lambda^{2} \mu_{\mathrm{E}} / Q$ increases monotonically with a decrease in $\kappa a$ (or with an increase in the double-layer overlap) as $\varphi=0$, but may have a maximum at some finite value 


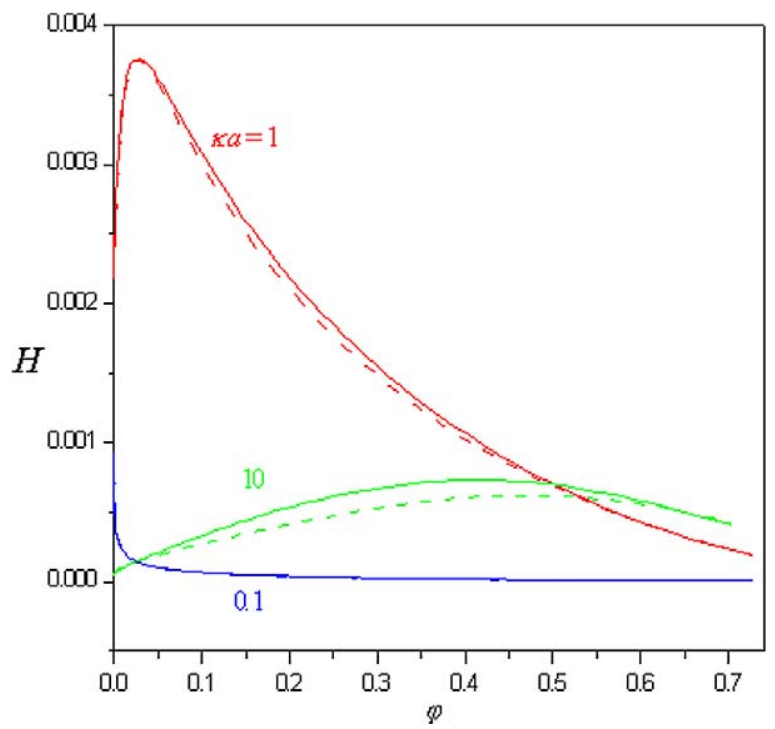

(a)

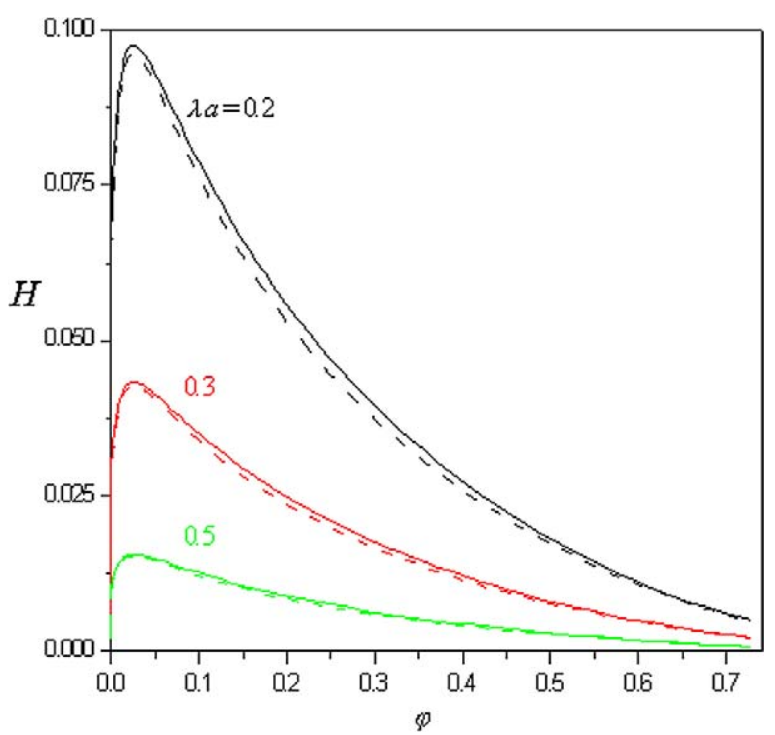

(b)

Fig. 4. Plots of the dimensionless coefficient $H$ in Eq. (26) for settling charged porous spheres in the $\mathrm{KCl}$ solution versus the parameter $\varphi$ : (a) $\lambda a=1$; (b) $\kappa a=1$. The solid and dashed curves represent the calculations for the Happel and Kuwabara cell models, respectively.

of $\kappa a$ and approaches zero in proportion to $(\kappa a)^{2}$ in the limit $\kappa a \rightarrow 0$ for any finite value of $\varphi$; the location of this maximum again shifts to greater $\kappa a$ as $\varphi$ increases, as shown in Fig. 5. For any values of $\lambda a$ and $\varphi, \eta \lambda^{2} \mu_{\mathrm{E}} / Q$ equals unity in the limit $\kappa a \rightarrow \infty$. For fixed values of $\kappa a$ and $\varphi, \eta \lambda^{2} \mu_{\mathrm{E}} / Q$ increases monotonically with an increase in $\lambda a$, approaches a constant value (e.g., $\eta \lambda^{2} \mu_{\mathrm{E}} / Q=1$ as $\varphi=0$ ) in the limit $\lambda a \rightarrow 0$, and is proportional to $(\lambda a)^{2}$ in the limit $\lambda a \rightarrow \infty$. For specified values of $\kappa a$ and $\lambda a$ in a broad range, $\eta \lambda^{2} \mu_{\mathrm{E}} / Q$ is a monotonic decreasing function of $\varphi$. Figs. 5-7 indicate that, for any combination of $\kappa a, \lambda a$, and $\varphi$, the Kuwabara model predicts a smaller value for the dimensionless sedimentation potential than the Happel model does, but the difference in general is negligible.

In a previous analysis for the sedimentation velocity and potential in suspensions of identical charged soft particles through

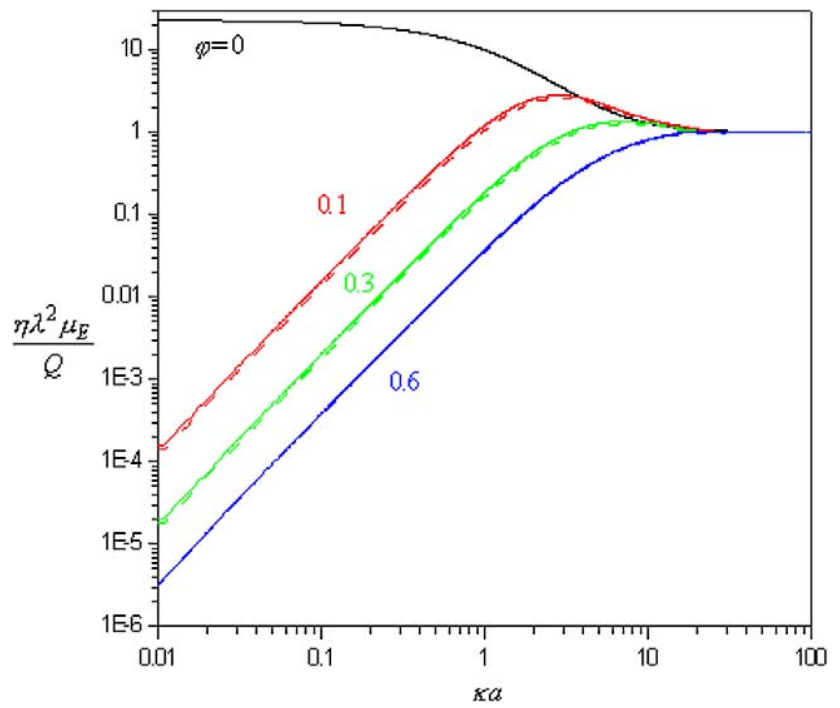

(a)

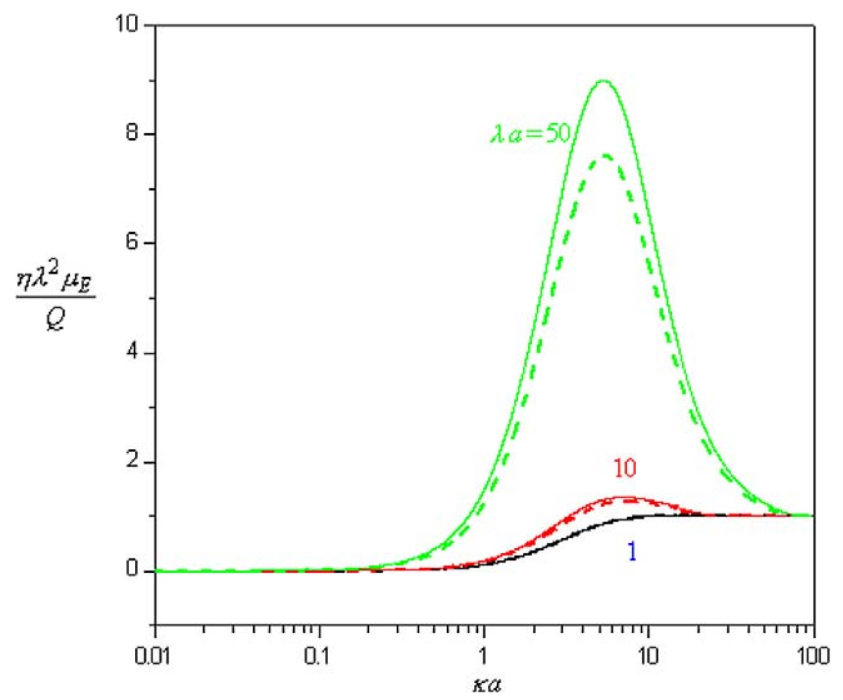

(b)

Fig. 5. Plots of the dimensionless sedimentation potential $\eta \lambda^{2} \mu_{\mathrm{E}} / Q$ in a suspension of identical charged porous spheres calculated from Eq. (30) versus the parameter $\kappa a$ : (a) $\lambda a=10$; (b) $\varphi=0.3$. The solid and dashed curves represent the calculations for the Happel and Kuwabara cell models, respectively.

the use of the Kuwabara cell model, it was assumed that the effects of the overlap of the electric double layers of adjacent particles and of the relaxation of the diffuse ions in the double layers could be neglected [31]. Comparisons of the relevant results of the dimensionless coefficients $H$ and $\eta \lambda^{2} \mu_{\mathrm{E}} / Q$ obtained in this previous analysis with our calculations relaxing these assumptions indicate that the errors in the sedimentation velocity and sedimentation potential in suspensions owing to neglect of these effects can be quite significant under typical conditions, even when the double layers are thin relative to the radius of the particles.

\section{Concluding remarks}

In this work, the steady-state sedimentation phenomena in a homogeneous suspension of identical charged porous spheres 


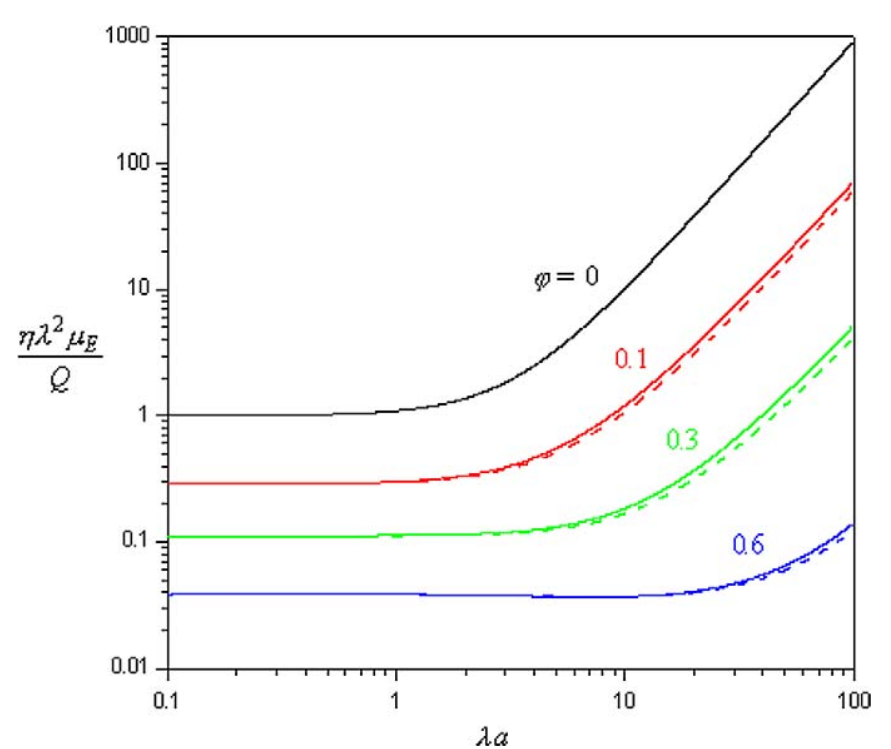

(a)

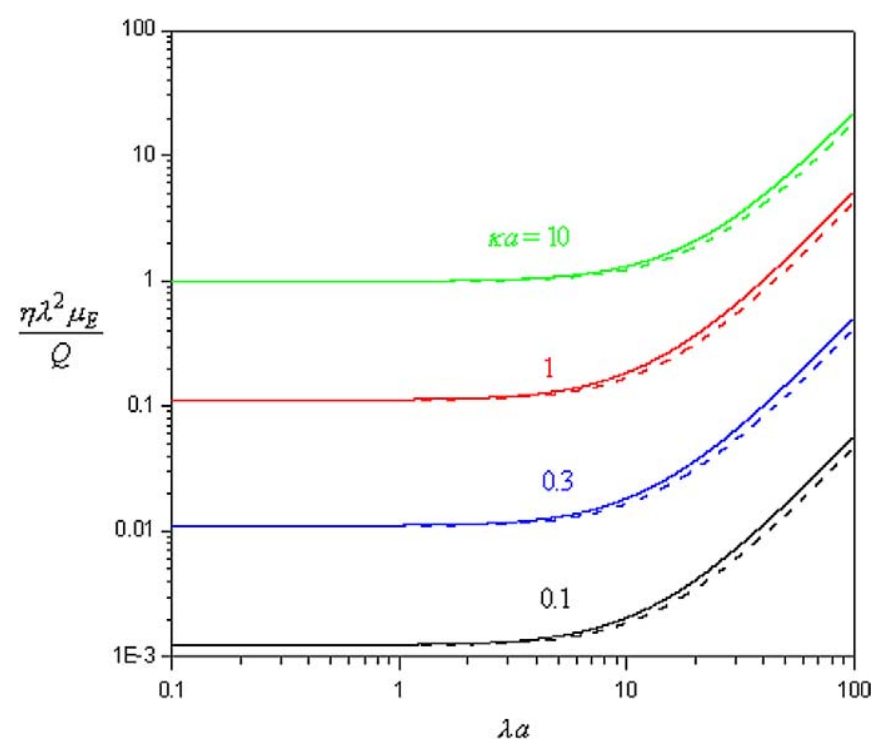

(b)

Fig. 6. Plots of the dimensionless sedimentation potential $\eta \lambda^{2} \mu_{\mathrm{E}} / Q$ in a suspension of identical charged porous spheres calculated from Eq. (30) versus the parameter $\lambda a$ : (a) $\kappa a=1$; (b) $\varphi=0.3$. The solid and dashed curves represent the calculations for the Happel and Kuwabara cell models, respectively.

in an electrolyte solution with arbitrary values of $\kappa a, \lambda a$, and $\varphi$ are analyzed by employing the Happel and Kuwabara cell models. Solving the linearized electrokinetic equations applicable to the system of a porous sphere in a unit cell by a regular perturbation method, we have obtained the ion concentration (or electrochemical potential energy) distributions, the electric potential profile, and the fluid flow field for the case of low electric potentials. The requirement that the total force exerted on the particle is zero leads to an explicit formula, Eq. (26), for the settling velocity of the particles. The effect of the particle charges is to reduce the settling velocity and the correction begins at the second order $Q^{2}$. Numerical results indicate that, for given values of $\lambda a$ and $\varphi$, this effect has a maximum at some finite values of $\kappa a$ and disappears when $\kappa a$ approaches

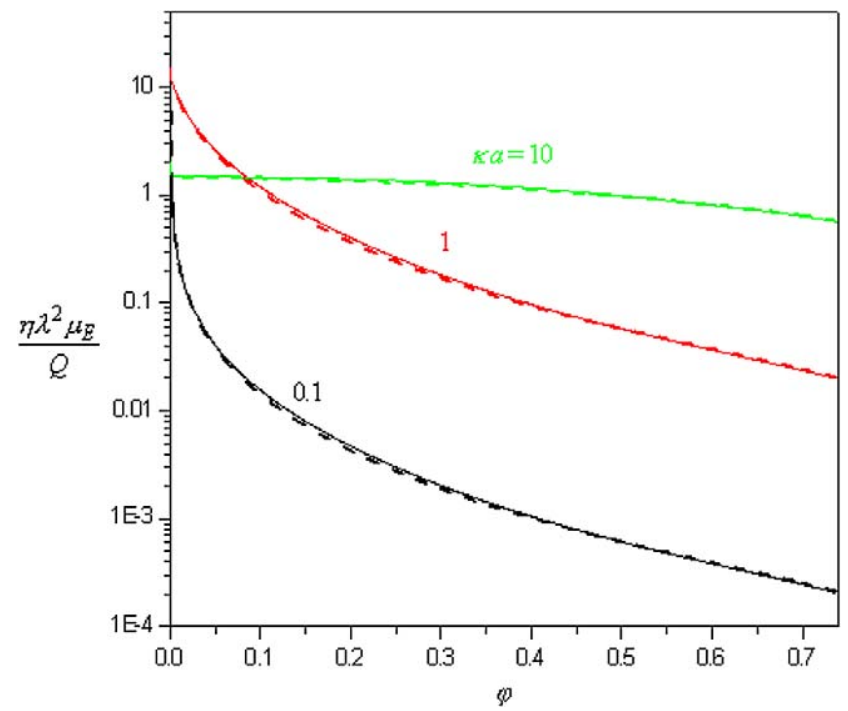

(a)

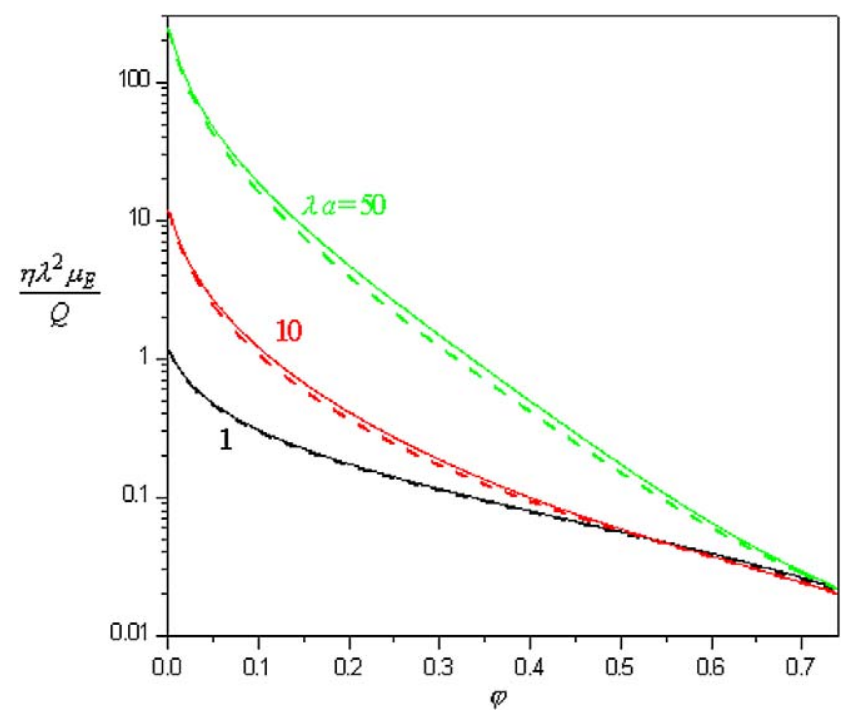

(b)

Fig. 7. Plots of the dimensionless sedimentation potential $\eta \lambda^{2} \mu_{\mathrm{E}} / Q$ in a suspension of identical charged porous spheres calculated from Eq. (30) versus the parameter $\varphi$ : (a) $\lambda a=10$; (b) $\kappa a=1$. The solid and dashed curves represent the calculations for the Happel and Kuwabara cell models, respectively.

zero and infinity. For fixed values of $\kappa a$ and $\varphi$, this effect increases monotonically with a decrease in $\lambda a$. Another explicit formula, Eq. (29), for the sedimentation potential is derived to the order $Q$ by letting the net electric current in the suspension be zero. The normalized sedimentation potential is found to increase with an increase in $\lambda a$ for specified values of $\kappa a$ and $\varphi$, and to decrease with an increase in $\varphi$ for given values of $\kappa a$ and $\lambda a$.

Equations (25)-(27), (29), and (30) are obtained on the basis of the Debye-Huckel approximation for the equilibrium potential distribution around the charged porous sphere in a unit cell. A similar formula for the sedimentation velocity of an impermeable sphere with a low zeta potential in an unbounded electrolyte solution was shown to give an excellent approximation for the case of reasonably high zeta potential (with an error 
less than $0.1 \%$ for $|\zeta| e / k T \leqslant 2$ in a $\mathrm{KCl}$ solution) [9]. Therefore, our results might be used tentatively for the situation of reasonably high electric potentials.

A limitation in both Booth's [1] classical theory and our perturbation analysis is that they are valid only for the case of a sufficiently small Péclet number. For the case of a relatively large Péclet number, however, Derjaguin and Dukhin $[6,45]$ obtained a fundamental result for the sedimentation potential of a dilute suspension of impermeable charged spheres and found that the Smoluchowski theory is still valid in spite of the double-layer polarization.

\section{Acknowledgment}

This research was partially supported by the National Science Council of the Republic of China.

\section{Appendix A. Definitions of some functions in Sections 3 and 5}

For conciseness the definitions of some functions in Sections 3 and 5 are listed here. In Eq. (17),

$$
\begin{aligned}
F_{i}(r)= & C_{i 1}+C_{i 2}\left(\frac{a}{r}\right)^{3} \alpha(\lambda r) \\
& +\delta_{i 2} \frac{2}{3 \lambda^{2}}\left[\int_{0}^{r} G(r) \mathrm{d} r-\frac{1}{r^{3}} \int_{0}^{r} r^{3} G(r) \mathrm{d} r\right. \\
& +\frac{3 \alpha(\lambda r)}{\lambda^{3} r^{3}} \int_{0}^{r} \beta(\lambda r) G(r) \mathrm{d} r \\
& \left.-\frac{3 \beta(\lambda r)}{\lambda^{3} r^{3}} \int_{0}^{r} \alpha(\lambda r) G(r) \mathrm{d} r\right]^{\text {for } 0<r<a,} \\
F_{i}(r)= & C_{i 3}+C_{i 4}\left(\frac{a}{r}\right)+C_{i 5}\left(\frac{a}{r}\right)^{3}+C_{i 6}\left(\frac{r}{a}\right)^{2} \\
& -\delta_{i 2} \frac{1}{15}\left[r^{2} \int_{0}^{r} G(r) \mathrm{d} r-5 \int_{0}^{r} r^{2} G(r) \mathrm{d} r\right. \\
& \left.+\frac{5}{r} \int_{0}^{r} r^{3} G(r) \mathrm{d} r-\frac{1}{r^{3}} \int_{0}^{r} r^{5} G(r) \mathrm{d} r\right] \\
\text { for } a< & <b, \quad r
\end{aligned}
$$

where $i=0$ and 2. In the above equations, $\delta_{i j}$ is the Kronecker delta which equals unity if $i=j$ but vanishes otherwise,

$$
\begin{aligned}
& G(r)=\frac{\varepsilon \kappa^{2}}{8 \pi \eta r}\left[F_{+}(r)-F_{-}(r)\right] \frac{\mathrm{d} \psi_{\mathrm{eq} 1}}{\mathrm{~d} r}, \\
& \alpha(x)=x \cosh x-\sinh x, \\
& \beta(x)=x \sinh x-\cosh x, \\
& C_{01}=\frac{-6\left[A_{2}+(\lambda a)^{2} \alpha(\lambda a)\right] \varphi^{-1 / 3}-3(\lambda a)^{2} \alpha(\lambda a) \varphi^{-2}}{W_{\mathrm{H}}},
\end{aligned}
$$

$$
\begin{aligned}
& C_{02}=\frac{6(\lambda a)^{2}\left(\varphi^{-1 / 3}-\varphi^{-2}\right)}{W_{\mathrm{H}}}, \\
& C_{03}=\frac{-3\left(2 A_{2}+A_{3}\right) \varphi^{-1 / 3}-(\lambda a)^{2} A_{1} \varphi^{-2}}{W_{\mathrm{H}}}, \\
& C_{04}=\frac{2(\lambda a)^{2} A_{2} \varphi^{-1 / 3}+3(\lambda a)^{4} \alpha(\lambda a) \varphi^{-2}}{W_{\mathrm{H}}}, \\
& C_{05}=\frac{-(\lambda a)^{2} A_{4} \varphi^{-2}}{W_{\mathrm{H}}}, \\
& C_{06}=\frac{(\lambda a)^{2} A_{4} \varphi^{-1 / 3}}{W_{\mathrm{H}}}
\end{aligned}
$$

for the Happel model, and

$$
\begin{aligned}
& C_{01}=\frac{-15 \alpha(\lambda a)\left(2 \varphi^{-1}+\varphi^{-2}\right)}{W_{\mathrm{K}}}, \\
& C_{02}=\frac{30\left(\varphi^{-1}-\varphi^{-2}\right)}{W_{\mathrm{K}}}, \\
& C_{03}=\frac{-5\left(A_{4} \varphi^{-1}+A_{1} \varphi^{-2}\right)}{W_{\mathrm{K}}}, \\
& C_{04}=\frac{15(\lambda a)^{2} \alpha(\lambda a) \varphi^{-2}}{W_{\mathrm{K}}}, \\
& C_{05}=\frac{2 A_{2} \varphi^{-1}-5 A_{4} \varphi^{-2}}{W_{\mathrm{K}}}, \\
& C_{06}=\frac{3 \alpha(\lambda a)(\lambda a)^{2} \varphi^{-1}}{W_{\mathrm{K}}}
\end{aligned}
$$

for the Kuwabara model, where

$$
\begin{aligned}
W_{\mathrm{H}}= & (\lambda a)^{2} A_{1} \varphi^{-2}-3(\lambda a)^{4} \alpha(\lambda a) \varphi^{-5 / 3} \\
& +3\left(2 A_{2}+A_{3}\right) \varphi^{-1 / 3}-2(\lambda a)^{2} A_{2}, \\
W_{\mathrm{K}}= & 5 A_{1} \varphi^{-2}-18(\lambda a)^{2} \alpha(\lambda a) \varphi^{-5 / 3} \\
& +10 A_{4} \varphi^{-1}-2 A_{2}, \\
A_{1}= & 3 \alpha(\lambda a)+2(\lambda a)^{3} \cosh (\lambda a), \\
A_{2}= & 15 \alpha(\lambda a)+(\lambda a)^{3} \cosh (\lambda a)-6(\lambda a)^{2} \sinh (\lambda a), \\
A_{3}= & (\lambda a)^{2}\left[(\lambda a)^{2}+12\right] \alpha(\lambda a)-4(\lambda a)^{4} \sinh (\lambda a), \\
A_{4}= & 6 \alpha(\lambda a)+(\lambda a)^{3} \cosh (\lambda a)-3(\lambda a)^{2} \sinh (\lambda a) .
\end{aligned}
$$

The expressions for the coefficients $C_{2 j}$ are lengthy; the full versions are available in W.C. Chen's M.S. thesis [44] or on request from the corresponding author. In Eqs. (18), (19), and (A.2),

$$
\begin{aligned}
F_{ \pm}(r)= & \pm \frac{1}{3 D_{ \pm}}\left[\frac{2 r}{b^{3}} \int_{0}^{b} r^{3} F_{0}(r) \frac{\mathrm{d} \psi_{\mathrm{eq} 1}}{\mathrm{~d} r} \mathrm{~d} r\right. \\
& \left.+r \int_{r}^{b} F_{0}(r) \frac{\mathrm{d} \psi_{\mathrm{eq} 1}}{\mathrm{~d} r} \mathrm{~d} r+\frac{1}{r^{2}} \int_{0}^{r} r^{3} F_{0}(r) \frac{\mathrm{d} \psi_{\mathrm{eq} 1}}{\mathrm{~d} r} \mathrm{~d} r\right]
\end{aligned}
$$

$F_{\psi}(r)=\frac{1}{2 \kappa r^{2}}\left\{\frac{\left(\kappa^{2} b^{2}+2 \kappa b+2\right) e^{-\kappa b}}{(\kappa b)^{2} \sinh (\kappa b)-2 \alpha(\kappa b)}\right.$ 


$$
\begin{aligned}
& \times \alpha(\kappa r) \int_{0}^{b} \alpha(\kappa r)\left[F_{+}(r)-F_{-}(r)\right] \mathrm{d} r \\
& +\alpha(\kappa r) \int_{r}^{b}(\kappa r+1) e^{-\kappa r}\left[F_{+}(r)-F_{-}(r)\right] \mathrm{d} r \\
& \left.+(\kappa r+1) e^{-\kappa r} \int_{0}^{r} \alpha(\kappa r)\left[F_{+}(r)-F_{-}(r)\right] \mathrm{d} r\right\}
\end{aligned}
$$

In Eq. (30),

$$
\begin{aligned}
& L_{1}=\alpha(\kappa b)(\kappa a-1) e^{\kappa a}-\alpha(\kappa a)(\kappa b-1) e^{\kappa b}, \\
& L_{2}=\kappa b \cosh (\kappa a-\kappa b)+\sinh (\kappa a-\kappa b), \\
& L_{3}=\varphi^{1 / 3} \cosh (\kappa a-\kappa b)+\kappa a \sinh (\kappa a-\kappa b), \\
& B_{1}=2 A_{2} \varphi^{5 / 3}+3(\lambda a)^{2} \alpha(\lambda a), \\
& B_{2}=(\lambda a)^{2} A_{1}+3\left(2 A_{2}+A_{3}\right) \varphi^{5 / 3} .
\end{aligned}
$$

\section{References}

[1] F. Booth, J. Chem. Phys. 22 (1954) 1956.

[2] S.S. Dukhin, B.V. Derjaguin, in: E. Matijevic (Ed.), Surface and Colloid Science, vol. 7, Wiley, New York, 1974.

[3] R.J. Hunter, Zeta Potential in Colloid Science, Academic Press, London, 1981.

[4] D.A. Saville, Adv. Colloid Interface Sci. 16 (1982) 267.

[5] J.H. Masliyah, Electrokinetic Transport Phenomena, AOSTRA, Edmonton, Alberta, Canada, 1994.

[6] S.S. Dukhin, Adv. Colloid Interface Sci. 44 (1993) 1.

[7] D. Stigter, J. Phys. Chem. 84 (1980) 2758.

[8] P.H. Wiersema, A.L. Leob, J.Th.G. Overbeek, J. Colloid Interface Sci. 22 (1966) 78

[9] S.R. de Groot, P. Mazur, J.Th.G. Overbeek, J. Chem. Phys. 20 (1952) 1825.

[10] H. Ohshima, T.W. Healy, L.R. White, R.W. O’Brien, J. Chem. Soc. Faraday Trans. 2 (80) (1984) 1299.
[11] J.J. Hermans, H. Fujita, K. Ned. Akad. Wet. Proc. Ser. B 58 (1955) 182.

[12] J.J. Hermans, J. Polym. Sci. 18 (1955) 527.

[13] H.C. Brinkman, Appl. Sci. Res. A 1 (1947) 27.

[14] P. Debye, M. Bueche, J. Chem. Phys. 16 (1948) 573.

[15] N. Imai, K. Iwasa, Israel J. Chem. 11 (1973) 223.

[16] I. Noda, M. Nagasawa, M. Ota, J. Am. Chem. Soc. 86 (1964) 5075.

[17] H.J. Keh, Y.C. Liu, J. Colloid Interface Sci. 195 (1997) 169.

[18] Y.C. Liu, H.J. Keh, Colloids Surf. A 140 (1998) 245.

[19] Y.C. Liu, H.J. Keh, Langmuir 14 (1998) 1560.

[20] H. Ohshima, J. Colloid Interface Sci. 228 (2000) 190.

[21] R.J. Hill, D.A. Saville, W.B. Russel, J. Colloid Interface Sci. 258 (2003) 56.

[22] J.J. Lopez-Garcia, C. Grosse, J. Horno, J. Colloid Interface Sci. 265 (2003) 327.

[23] J. Happel, AIChE J. 4 (1958) 197.

[24] S. Kuwabara, J. Phys. Soc. Jpn. 14 (1959) 527.

[25] S. Levine, G. Neale, N. Epstein, J. Colloid Interface Sci. 57 (1976) 424.

[26] V.N. Shilov, N.I. Zharkikh, Yu.B. Borkovskaya, Colloid J. 43 (1981) 434.

[27] A.S. Dukhin, V. Shilov, Yu. Borkovskaya, Langmuir 15 (1999) 3452.

[28] H. Ohshima, J. Colloid Interface Sci. 208 (1998) 295.

[29] F. Carrique, F.J. Arroyo, A.V. Delgado, Colloids Surf. A 195 (2001) 157.

[30] H.J. Keh, J.M. Ding, J. Colloid Interface Sci. 227 (2000) 540.

[31] H. Ohshima, J. Colloid Interface Sci. 229 (2000) 140.

[32] J. Koplik, H. Levine, A. Zee, Phys. Fluids 26 (1983) 2864.

[33] K. Matsumoto, A. Suganuma, Chem. Eng. Sci. 32 (1977) 445.

[34] J.H. Masliyah, M. Polikar, Can. J. Chem. Eng. 58 (1980) 299.

[35] S. Kawahata, H. Ohshima, N. Muramatsu, T. Kondo, J. Colloid Interface Sci. 138 (1990) 182.

[36] K. Morita, N. Muramatsu, H. Ohshima, T. Kondo, J. Colloid Interface Sci. 147 (1991) 457.

[37] O. Aoyanagi, N. Muramatsu, H. Ohshima, T. Kondo, J. Colloid Interface Sci. 162 (1994) 222.

[38] G. Neale, N. Epstein, W. Nader, Chem. Eng. Sci. 28 (1973) 1865.

[39] V. Natraj, S.B. Chen, J. Colloid Interface Sci. 251 (2002) 200.

[40] J. Happel, H. Brenner, Low Reynolds Number Hydrodynamics, Nijhoff, Dordrecht, The Netherlands, 1983

[41] H.J. Keh, Y.C. Chang, Colloid Polym. Sci. 283 (2005) 627.

[42] T.G.M. van de Ven, Colloidal Hydrodynamics, Academic Press, London, 1989.

[43] S. Levine, G.H. Neale, J. Colloid Interface Sci. 47 (1974) 520.

[44] W.C. Chen, M.S. thesis, National Taiwan University, 2005.

[45] B.V. Derjaguin, S.S. Dukhin, in: B.V. Derjaguin (Ed.), Research in Surface Forces, vol. 3, Plenum, New York, 1971. 